\title{
Antimicrobial activity of cream incorporated with silver nanoparticles biosynthesized from Withania somnifera
}

This article was published in the following Dove Press journal:

International Journal of Nanomedicine

22 September 2015

Number of times this article has been viewed

\author{
Gregory Marslin' \\ Rajendran K Selvakesavan' \\ Gregory Franklin' \\ Bruno Sarmento 2,3 \\ Alberto CP Dias' \\ 'Centre for the Research and \\ Technology of Agro-Environment \\ and Biological Sciences (CITAB-UM), \\ AgroBioPlant Group, Department of \\ Biology, University of Minho, Braga, \\ Portugal; ' Instituto de Engenharia \\ Biomédica (INEB), University of Porto, \\ Porto, Portugal; ${ }^{3}$ CESPU, Instituto \\ Universitário de Ciências da Saúde, \\ Gandra, Portugal
}

Correspondence: Alberto CP Dias University of Minho, Campus de Gualtar, 47I 0-057 Braga, Portugal

$\mathrm{Tel}+35$ I 25360 I5I0

Fax +35I 253678980

Email acpdias@bio.uminho.pt
Abstract: We report on the antimicrobial activity of a cream formulation of silver nanoparticles (AgNPs), biosynthesized using Withania somnifera extract. Aqueous extracts of leaves promoted efficient green synthesis of AgNPs compared to fruits and root extracts of $W$. somnifera. Biosynthesized AgNPs were characterized for their size and shape by physical-chemical techniques such as UV-visible spectroscopy, laser Doppler anemometry, transmission electron microscopy, scanning electron microscopy, atomic force microscopy, X-ray diffraction, and X-ray energy dispersive spectroscopy. After confirming the antimicrobial potential of AgNPs, they were incorporated into a cream. Cream formulations of $\mathrm{AgNPs}$ and $\mathrm{AgNO}_{3}$ were prepared and compared for their antimicrobial activity against human pathogens (Staphylococcus aureus, Pseudomonas aeruginosa, Proteus vulgaris, Escherichia coli, and Candida albicans) and a plant pathogen (Agrobacterium tumefaciens). Our results show that AgNP creams possess significantly higher antimicrobial activity against the tested organisms.

Keywords: Withania somnifera, green synthesis, silver nanoparticles cream, antimicrobial activity

\section{Introduction}

Nanotechnology and nanotools have gained much attention due to their wide range of applications in physics, chemistry, biology, material science, and medicine. ${ }^{1}$ Metal nanoparticles like silver, gold, and copper have been used for diagnosis and treatment of disease because of their catalytic, optical, electronic, antimicrobial, and magnetic properties. ${ }^{2}$ Silver nanoparticles (AgNPs) are generally synthesized by physical and chemical methods such as electrochemical reduction and thermal evaporation, ${ }^{3-5}$ but these methods are time-consuming and difficult to scale up. Furthermore, these methods are environmentally unsafe due to the use of toxic chemicals. A method that could avoid all the above-mentioned limitations is the ideal choice for preparing AgNPs. Recently, it has been shown that several plant extracts can undergo highly controlled and hierarchical assembly, which makes them suitable for the development of a reliable and ecofriendly process for metal nanoparticle synthesis. ${ }^{6-11}$

Withania somnifera (L.) Dunal, (Solanaceae), commonly known as ashwagandha or winter cherry, is a well-known medicinal plant in Ayurvedic medicine. The principal active compounds include several withanolide-type compounds. ${ }^{12,13}$ Due to the nontoxic and high medicinal value of $W$. somnifera (WS), this plant is widely used all over the world. Roots, and less often leaves and fruits, have been used as phytomedicines in the form of decoction, infusions, ointment, powder, and syrup. ${ }^{12-14}$ Nowadays, this plant is cultivated as a crop to support the high demand of biomass and a sustainable quality for the needs 
of pharmaceutical industry. ${ }^{15}$ In this study, we biosynthesized AgNPs using WS extract and identified the compounds responsible for the formation of AgNPs. To demonstrate the potential pharmaceutical and industrial applications of the synthesized AgNPs, we developed a cream formulation for AgNPs and evaluated antimicrobial activity in a range of microorganisms.

\section{Materials and methods Preparation of WS plant extract}

WS plants were collected from Pondicherry Botanical Garden (Pondicherry, India). Leaves, fruits, and roots were dried under shade at $25^{\circ} \mathrm{C}$ and powdered. WS aqueous extracts $(2 \%, 4 \%, 6 \%$, and $8 \%, \mathrm{w} / \mathrm{v})$ were prepared by boiling leaves, fruits, and root biomass separately in $500 \mathrm{~mL}$ Erlenmeyer flasks containing $100 \mathrm{~mL}$ of sterile distilled water for 1 hour. Aqueous extracts were obtained by centrifugation at $10,000 \mathrm{rpm}$ for 10 minutes followed by filtration.

\section{Synthesis of silver nanoparticles}

We screened several WS extracts for the formation of AgNPs. First, $10 \mathrm{~mL}$ of $1 \mathrm{mM} \mathrm{AgNO}_{3}$ solution was added to $500 \mu \mathrm{L}$ aliquots of different concentrations $(2 \%, 4 \%, 6 \%$, and $8 \%$, $\mathrm{w} / \mathrm{v}$ ) of aqueous WS extracts of roots, leaves, and fruits. The reaction was allowed to take place in $25 \mathrm{~mL}$ volumetric flasks at room temperature; solutions were vortexed ( 5 minutes) thrice daily and continuously monitored for color changes, which is an indication for $\mathrm{AgNO}_{3}$ conversion into AgNPs. Simultaneously, aliquots were taken and analyzed for absorbance at 300-700 nm in a UV-visible (UV-vis) spectrophotometer. After the formation of AgNPs, the solution was centrifuged at 20,000 rpm for 15 minutes and the supernatants were discarded. Nanoparticles were washed with $10 \mathrm{~mL}$ of deionized water, centrifuged again, and the supernatant was discarded. Finally, AgNPs were redispersed in $10 \mathrm{~mL}$ of deionized water.

Since AgNPs were efficiently formed in $6 \% \mathrm{w} / \mathrm{v}$ aqueous leaf extract, we chose this extract for further standardization of $\mathrm{AgNO}_{3}$ concentration. Briefly, different concentrations of $\mathrm{AgNO}_{3}(0.1,0.5,1$, and $2 \mathrm{mM})$ were prepared and $10 \mathrm{~mL}$ of each concentration was added to $6 \% \mathrm{w} / \mathrm{v}$ leaf extract, and the nanoparticle formation was recorded. As the combination of $6 \%$ leaf extract and $1 \mathrm{mM} \mathrm{AgNO}_{3}$ was found to be the optimum, AgNPs synthesized with this combination were used in all further experiments.

\section{Physical characterization of AgNPs}

Particle size and zeta potential analysis

The size and zeta potential of AgNPs were determined by laser Doppler anemometry using a Zetasizer (Zetasizer nano
ZS; Malvern Instruments, Malvern, UK). Briefly, $100 \mu \mathrm{L}$ of nanoparticles was diluted to $1 \mathrm{~mL}$ with deionized water. An electric field of $150 \mathrm{mV}$ was applied to observe the electrophoretic velocity of the particles. ${ }^{16}$ All measurements were made at room temperature. Three independent samples were run, in triplicate.

\section{Electron microscopy analysis}

Morphological characterization of the AgNPs was done by transmission electron microscopy (TEM) and scanning electron microscopy (SEM) analysis. For the TEM analyses, the AgNPs were negatively stained with 1\% solution of sodium uranyl acetate and analyzed using TEM (JEOL-1400, 902A; JEOL, Tokyo, Japan). ${ }^{17}$ For the SEM analysis, samples were prepared by dropping nanoparticle suspension onto aluminum stubs and allowing them to air dry. The air-dried particles were sputter coated with gold under vacuum by using a Fiscon Instrument SC 502 and observed by SEM (Leica Cambridge S 360; Leica Microsystems, Wetzlar, Germany) for imaging. X-ray energy dispersive spectroscopy analysis was conducted with the same instrument to confirm the elemental composition of the sample.

\section{Atomic force microscopy}

The surface morphology of the AgNPs was studied by atomic force microscopy. A drop of the AgNPs was placed onto a $2 \times 2 \mathrm{~mm}^{2}$ glass slide and left until the liquid had evaporated. Images were recorded using tapping mode in air on a multimode NanoScope IIa instrument equipped with a J scanner (Veeco Instruments, Santa Barbara, CA, USA) and silicon nanoprobes (NCHV; Veeco Instruments). All images (600 nm wide) were fitted to a plane using the 1 degree flatten procedure included in the NanoScope software version $4.43 \mathrm{r} 8$.

\section{X-ray diffractometry analysis}

An X-ray diffraction study of AgNPs was performed using an X-ray diffractometer (Philips PW1710; Philips Co., Amsterdam, the Netherlands) with a horizontal goniometer. The samples were placed in the sample holder and scanned at a rate of $1^{\circ}$ per minute from $0^{\circ}$ to $70^{\circ}$.

\section{Chemical characterization of AgNPs}

AgNPs and leaf aqueous extracts of WS were analyzed by high-performance liquid chromatography with diode array detector (HPLC-DAD) (Merck Hitachi LaChrom Elite; Merck KGaA, Darmstadt, Germany). ${ }^{18}$ Briefly, $2 \mathrm{~mL}$ of AgNPs were centrifuged and the pelleted AgNPs were 
extracted with $1 \mathrm{~mL}$ of $50 \%$ methanol. The extract solution of AgNPs and leaf extracts of WS were filtered (Nylaflo membrane $0.45 \mu \mathrm{M}$ ) prior to the HPLC analysis. Aliquots of these samples $(20 \mu \mathrm{L})$ were directly injected in HPLC system. Chromatographic separations were carried out at $30^{\circ} \mathrm{C}$ on a LiChrospher RP-18e column (Merck KGaA) using $0.1 \%$ acetic acid in water (solution $\mathrm{A}$ ) and $0.1 \%$ acetic acid in acetonitrile (solution B) as the mobile phases at a flow rate of $0.7 \mathrm{~mL} / \mathrm{min}$. The following gradient was used: from 0 to 30 minutes, mobile phase was decreased from $55 \%$ to $10 \%$ of A. Data were recorded in the range $200-520 \mathrm{~nm}$, and chromatograms were recorded at 235 and $350 \mathrm{~nm}$. Compounds present in the sample were quantified by using external standards of catechin, p-coumaric acid, luteolin7-glucoside (Sigma-Aldrich Co., St Louis, MO, USA), and withanolides (Natural Remedies, Bangalore, India).

\section{Antimicrobial activity studies}

Screening of $\mathrm{AgNO}_{3}$,WS leaf extract, and AgNPs for antimicrobial activity

The preliminary antibacterial potential of AgNPs and the components used in the synthesis $\left(\mathrm{AgNO}_{3}\right.$ and WS leaf extract) was tested against Escherichia coli by disc diffusion method. Briefly, $100 \mu \mathrm{L}$ aliquots of $E$. coli culture were spread on Petri dishes containing agar-solidified Luria broth (LB) medium. Sterile paper discs $(5 \mathrm{~mm}$ diameter) impregnated with equivalent concentrations of AgNPs $(20 \mu \mathrm{L})$, WS leaf extract, and $\mathrm{AgNO}_{3}$ solution were placed on the medium. Paper discs impregnated with water $(20 \mu \mathrm{L})$ were used as control. The plates were incubated at $37^{\circ} \mathrm{C}$ for 24 hours and the inhibition zones were measured.
Cream preparation using AgNPs and its antimicrobial activity

Cream formulation with AgNPs was prepared with Croda Base CR2 (EMFAL, MG, Brazil). Briefly, CR2 was prepared at a concentration of $15 \%(\mathrm{w} / \mathrm{v})$ in ultrapure water (control) or in a solution containing $1 \% \mathrm{AgNO}_{3}\left(\mathrm{AgNO}_{3}\right.$ cream) or equivalent AgNPs (AgNPs cream).

The antimicrobial activities of the creams were evaluated by colony forming unit method. Briefly, to $950 \mu \mathrm{L}$ of grown microbial cultures (Staphylococcus aureus, Pseudomonas aeruginosa, Candida albicans, Proteus vulgaris, E. coli, Agrobacterium tumefaciens), $50 \mu \mathrm{L}$ of cream $\left(\mathrm{AgNO}_{3}\right.$, AgNPs, or cream containing only water) was added at various dilutions and kept at $37^{\circ} \mathrm{C}$. Cultures with $50 \mu \mathrm{L}$ of water served as positive control. After 24 hours, $100 \mu \mathrm{L}$ from each treatment and control was plated on LB agar medium using a sterile T-spreader. The colony forming units in each plate were counted after overnight incubation. All the experiments were done in quadruplicate.

\section{Results and discussion}

\section{Green synthesis of silver nanoparticles}

Among the different concentrations of WS extracts and $\mathrm{AgNO}_{3}$ used for the synthesis of nanoparticles, $6 \% \mathrm{w} / \mathrm{v} \mathrm{WS}$ and $1 \mathrm{mM} \mathrm{AgNO}_{3}$ showed better reaction profiles and were selected for further studies. When $\mathrm{AgNO}_{3}$ was added, leaf aqueous extract changed into reddish color (Figure 1A), whereas fruit and root extracts did not change their color within 7 days. UV-vis absorbance at $430 \mathrm{~nm}$ confirmed the reduction of silver ion to silver metal due to the reaction with the components present in the aqueous leaf extract of WS (Figure 1B). It was evident that the WS leaf extract
A

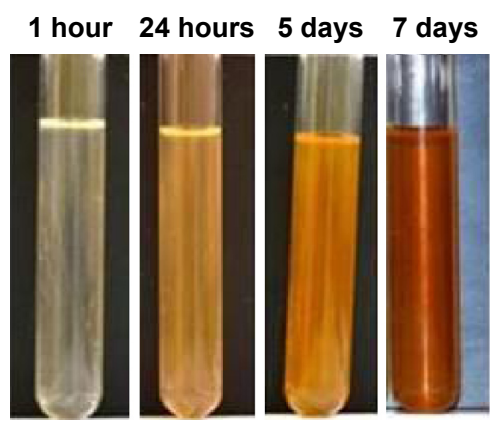

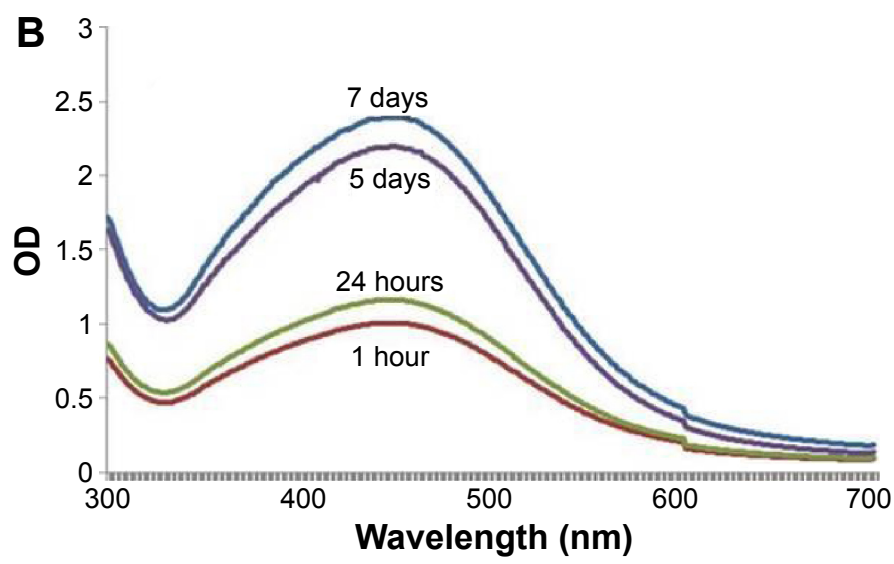

Figure I Biosynthesis of AgNPs.

Notes: Photograph showing the formation of AgNPs using Withania somnifera extract and visual observation of color changes (A). UV-vis spectra of AgNPs synthesized using $6 \% \mathrm{w} / \mathrm{w}$ Withania somnifera aqueous extract with $\mathrm{I} \mathrm{mM} \mathrm{AgNO}_{3}$ at different time intervals (B).

Abbreviations: AgNPs, silver nanoparticles; OD, optical density; UV-vis, ultraviolet-visible. 
contains compounds suitable for green synthesis, whereas root and fruit extracts do not. Recently, the photosensitized synthesis of AgNPs via using WS leaf powder was reported. ${ }^{19}$

\section{Characterization of AgNPs}

Particle size and size distribution are important characteristics of nanoparticles, as they determine their biological fate, toxicity, targeting ability, and stability. The synthesized AgNPs had a size ranging between 70 and $110 \mathrm{~nm}$, as confirmed by laser Doppler anemometry analysis (Figure 2A). The zeta potential was found to be $-30.0 \pm 1.8 \mathrm{mV}$, indicating the stability of the formulation.
The TEM, atomic force microscopy, and SEM images of AgNPs correlate with dynamic light scattering data and showed particles with a spherical shape (Figure 2B-E). AgNPs were brighter and easily identified by backscattered light in SEM images, since metals like silver show higher secondary electron emission (Figure 2D-E). X-ray energy dispersive spectroscopy spectrum of synthesized AgNPs (Figure 2F) clearly exhibited the presence of elemental silver metal. The sharp signal peak of silver confirmed the reduction of silver nitrate to silver by nanoparticles.

The crystalline nature of AgNPs was confirmed by X-ray diffraction analysis (Figure 3). The intensity of peaks reflects the high degree of crystallinity of the AgNPs. The distinct
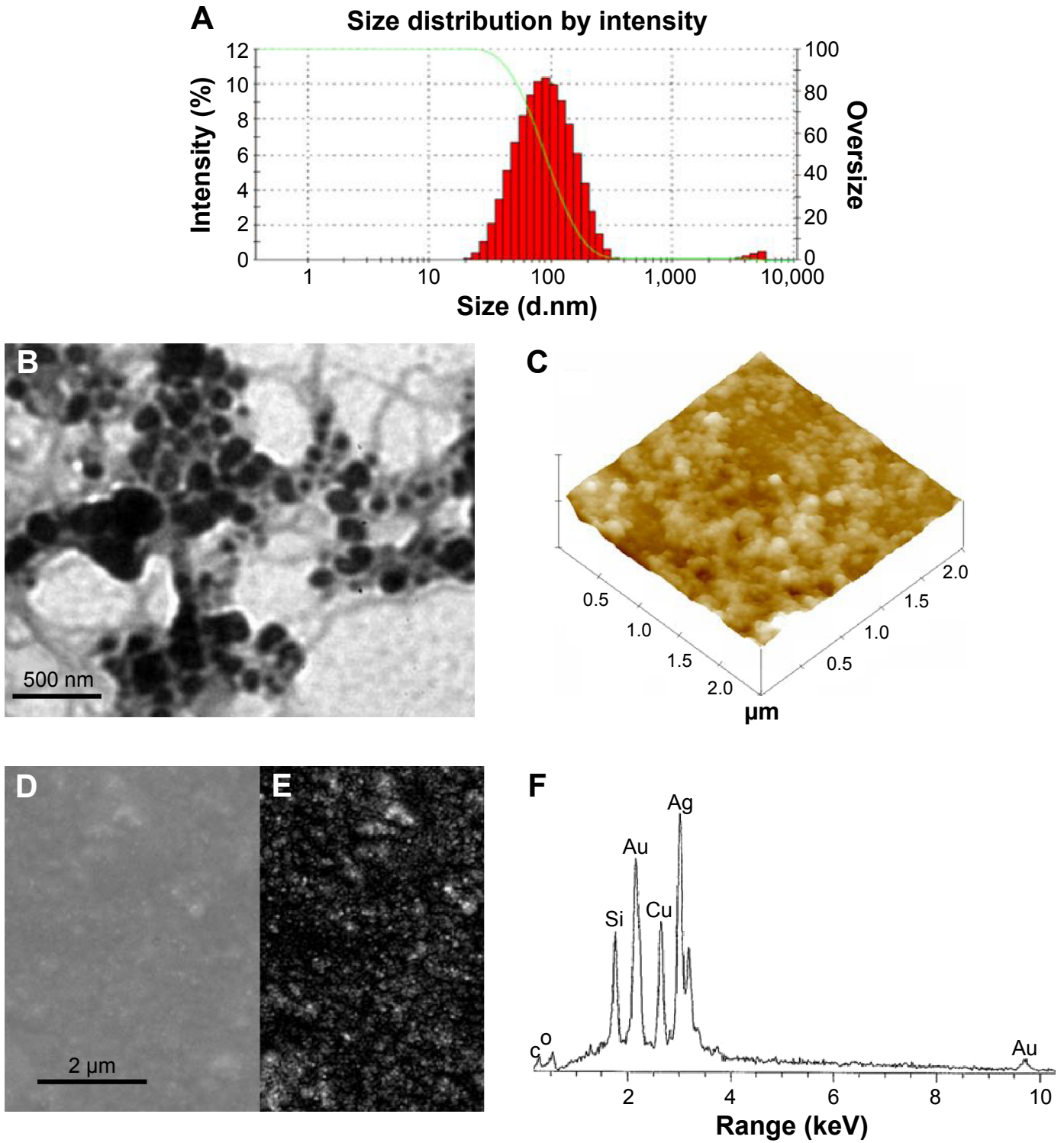

Figure 2 Morphological characterization of AgNPs.

Notes: Particle size distribution (A), TEM image (B), AFM height image (C), SEM primary image (D), backscattered image (E), and X-ray energy dispersive spectrum (F), of AgNPs.

Abbreviations: TEM, transmission electron microscopy; AgNPs, silver nanoparticles; AFM, atomic force microscopy; SEM, scanning electron microscopy. 


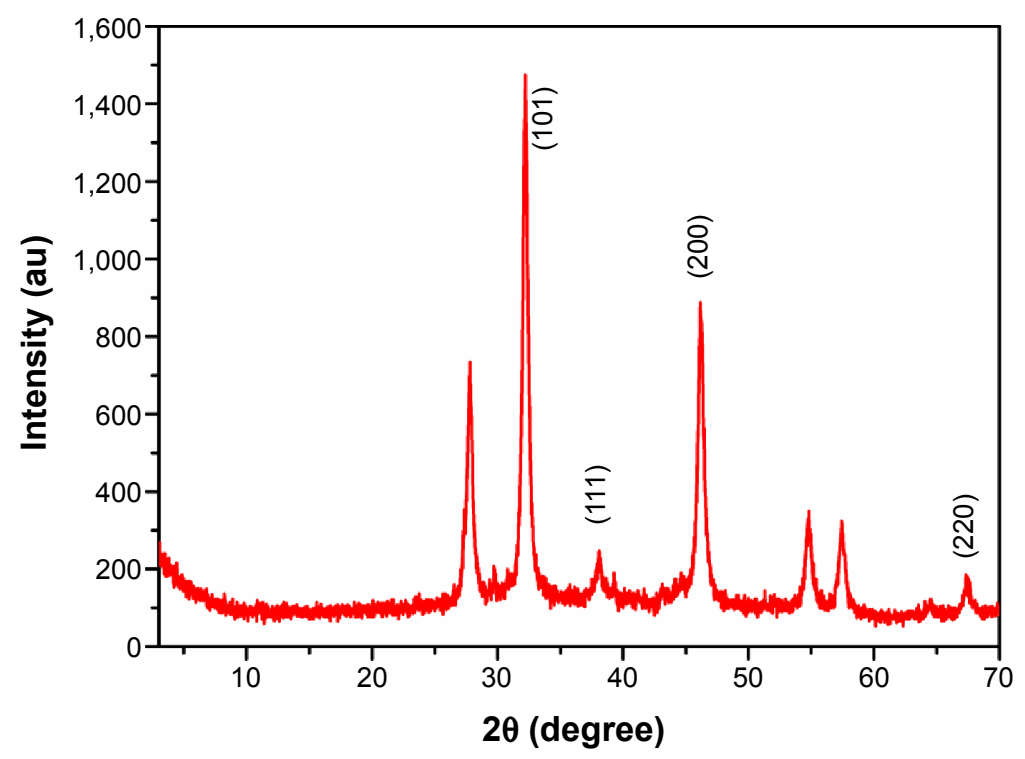

Figure 3 XRD patterns of the synthesized AgNPs with aqueous leaf extract of Withania Somnifera. Abbreviations: XRD, X-ray diffraction; AgNPs, silver nanoparticles.

diffraction peaks at $38.06,44.23$, and 67.43 were indexed with planes (111), (200), and (220). The intense X-ray diffraction pattern clearly showed the formation of AgNPs (JCPDS card no 04-0783, 1991). ${ }^{20}$ Amutha et al reported the same pattern of diffraction peak at 32.16 and indexed with plane (101) in biosynthesized AgNPs at room temperature. In the same way, we got a peak at 32.20 which was indexed with plane (101). ${ }^{21}$

\section{WS compounds responsible for green synthesis of AgNPs}

Although green synthesis of AgNPs has been reported using extracts from different plant species, the knowledge concerning the phytocompounds responsible for green synthesis is still lacking. It was proposed that phenolics like catechin may act as a reducing, stabilizing, and capping agent. ${ }^{22,23}$

In order to identify the WS compounds participating in green synthesis of AgNPs, we performed HPLC analysis. We identified several withanolides in WS leaf extract based on their characteristic UV-vis spectra (Figure 4A-D; Table 1). In addition, HPLC analysis of AgNPs revealed that some of the phenolic compounds present in the WS leaf extract were selectively trapped in the nanoparticles (Figure 4C and D). These compounds, based on their characteristic UV-vis spectra and further confirmation by coeluting with pure standards, were identified as catechin, p-coumaric acid, and luteolin-7glucoside (Figure 4C and D and Table 1). These substances were masked by other major compounds (peaks) present in the WS extract, namely withanolides; the compounds catechin, p-coumaric acid, and luteolin-7-glucoside were noticed in chromatograms of AgNPs as they were selectively trapped in AgNPs, and the other compounds were removed when the nanoparticles were washed. In addition, a major compound (peak 4) was found in AgNPs that was not identified in the original WS leaf aqueous extract. This compound had UV-vis spectra similar to other withanolides found in the leaf aqueous extract (peaks W2, W5, and W6) but with a different retention time and showing a bathochromic effect on its spectra. Taking this into consideration, this compound might be a derivative that originated from the interaction of some withanolide derivative with silver ions. So, we can conclude that the formation of AgNPs involved the interaction of silver ions with both selected phenolics and withanolides present in the WS aqueous leaf extract. Recently, the relevance of phenolic compounds for the green synthesis of metal nanoparticles from Eucalyptus globulus bark was reported. ${ }^{24}$

\section{Antimicrobial activity of AgNPs}

It is well known that a number of chemical forms of silver exhibit antimicrobial activities. ${ }^{25}$ AgNPs showed a wider bacterial inhibition zone than $\mathrm{AgNO}_{3}$ in all the analyzed bacteria ( $E$. coli, $P$. aeruginosa, and A. tumefaciens) (Figure 5A-C). The interaction of AgNPs with E. coli cells were studied using SEM analysis (Figure 6). The AgNPs treated cells ( 2 hours and 4 hours) showed increased disruption in the cell wall of $E$. coli cells with increased time interval. No disruption was seen in untreated cells, clearly indicating the enhanced antibacterial activity of AgNPs (Figure 6A-C). The results from colony counting method showed that the AgNPs significantly reduced the number of $E$. coli cells from $10^{6}$ to $1.3 \pm 0.9 \mathrm{CFUs} / \mathrm{mL}$, while the 

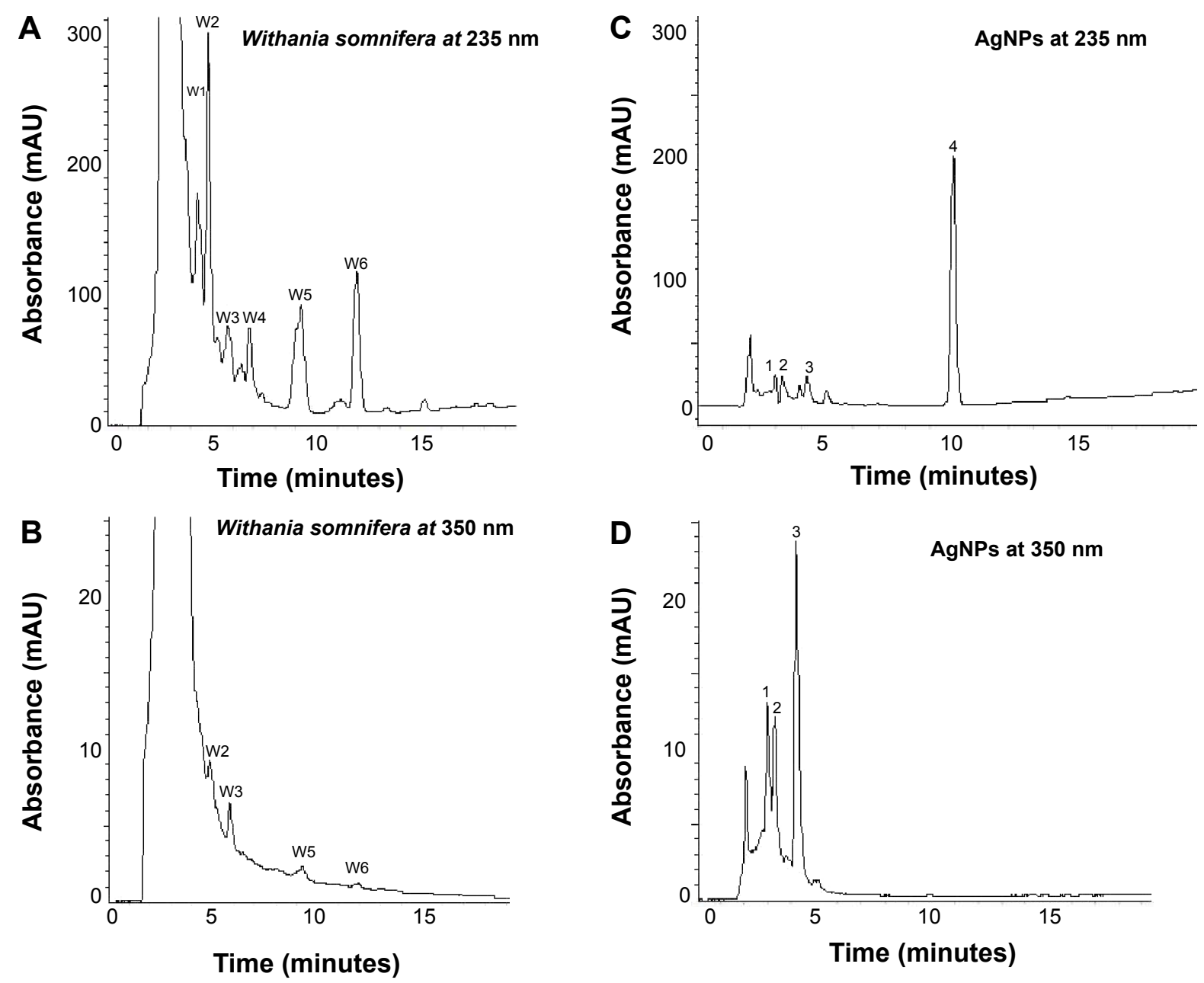

Figure 4 HPLC chromatograms of aqueous leaf extract of Withania somnifera and green synthesized AgNPs.

Notes: (A and B) HPLC chromatogram of aqueous leaf extract of Withania somnifera recorded at 235 and $350 \mathrm{~nm}$. WI-W6 are whitanolide-type compounds. (C and D) HPLC chromatogram of AgNPs recorded at 235 and $350 \mathrm{~nm}$. Peak I indicates catechin; peak 2 indicates p-coumaric acid; peak 3 indicates luteolin-7-glucoside; and peak 4 indicates a whitanolide-type compound.

Abbreviations: AgNPs, silver nanoparticles; HPLC, high-performance liquid chromatography.

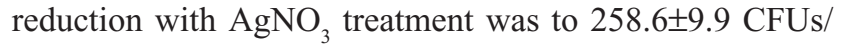
$\mathrm{mL}$, and no reduction was observed with WS aqueous extract treatment (Figure 7). These results further substantiate the higher antimicrobial activity ( 200 times more effective)

Table I Retention time of the compounds present in Withania somnifera aqueous leaf extract and AgNPs

\begin{tabular}{llll}
\hline Peak & \multicolumn{2}{l}{ Retention time (minutes) } & Compound \\
\cline { 2 - 3 } number & Aqueous leaf extract & AgNPs & \\
\hline WI & 4.32 & - & Withanolide \\
W2 & 4.82 & - & Withanolide \\
W3 & 5.74 & - & Withanolide \\
W4 & 6.82 & - & Withanolide \\
W5 & 9.26 & - & Withanolide \\
W6 & II.94 & - & Withanolide \\
I & - & 2.98 & Catechin \\
2 & - & 3.29 & P-coumaric acid \\
3 & - & 4.23 & Luteolin-7-glucoside \\
4 & - & 9.89 & Withanolide \\
\hline
\end{tabular}

Abbreviations: AgNPs, silver nanoparticles; W, whitanolide. of AgNPs compared with equivalent amounts of $\mathrm{AgNO}_{3}$. It has been reported that AgNPs show higher activity then their $\mathrm{AgNO}_{3}$ counterparts. ${ }^{26}$ Moreover, AgNPs biosynthesized from plant extracts showed enhanced antibacterial activity compared with chemically synthesized AgNPs. ${ }^{27}$

The lethality of AgNPs can occur by mechanisms such as damaging bacterial cellular proteins, disruption of cell membrane, blocking the microbial respiratory chain system, and penetration of nanoparticles into the cytoplasm. ${ }^{28,29}$ The higher antibacterial activity of $\mathrm{AgNPs}$, compared with $\mathrm{AgNO}_{3}$, could also be due to the presence of WS phenolics in the nanoparticles. It is well known that some phenolics possess antimicrobial activities or potentiate the activity of antibiotics, increasing their efficacy. Compounds like flavones and catechin derivatives, structurally similar to the ones identified in WS and AgNPs, have been shown to exhibit little antimicrobial activity. ${ }^{30,31}$ Several tea catechins in combination with oxacillin ( $\beta$-lactam antibiotic) showed an increase 

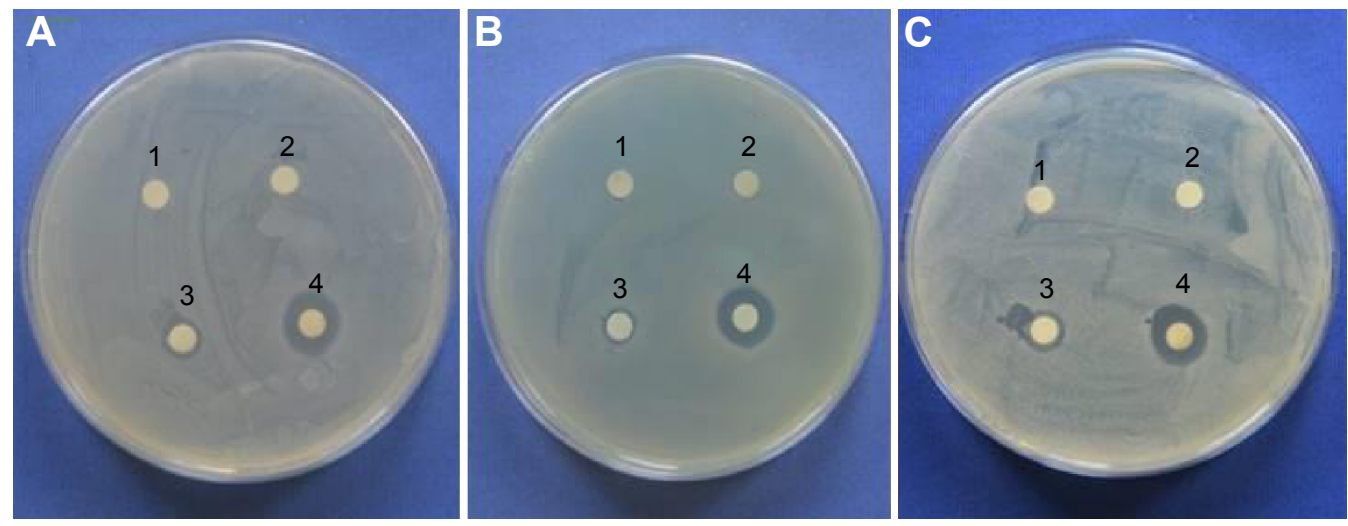

Figure 5 Antibacterial activity of AgNPs analyzed by disc diffusion method.

Notes: Bacterial growth without treatments (I) or treated with equivalent amount of Withania somnifera aqueous extract (2), $\mathrm{AgNO}_{3}(3)$, and $\mathrm{AgNPs}_{3}(4)$. The bacteria tested were Escherichia coli (A), Pseudomonas aeruginosa (B), and Agrobacterium tumefaciens (C).

Abbreviation: AgNPs, silver nanoparticles.

inactivity up to 128 -fold against several strains of S. aureus. ${ }^{31}$ Some antimicrobial mechanisms of the phenolics are related to efflux pump inactivation and cytoplasmic membrane destabilization, ${ }^{30}$ which might explain the observed effects (SEM analysis) of AgNPs in bacterial cells (Figure 6) and their higher activity compared with $\mathrm{AgNO}_{3}$ or WS extract alone (Figure 7).

Antibiotic creams are used to speed up the healing of wounds and prevent infection. Antibacterial activity of creams incorporated with $\mathrm{AgNO}_{3}$ and $\mathrm{AgNPs}$ were compared with an empty cream, against pathogenic clinical microbial isolates of $S$. aureus, $P$. aeruginosa, $C$. albicans, $P$. vulgaris, $E$. coli, and the plant pathogen $A$. tumefaciens. The results showed that the cream with AgNPs significantly reduced the number of colonies against all the tested pathogens compared to the cream containing $\mathrm{AgNO}_{3}$ (Table 2). These results further substantiate the higher antimicrobial activity of AgNPs cream against the Gram-positive bacteria, S. aureus ( 200 times more effective), compared with $\mathrm{AgNO}_{3}$ cream. Similarly, Gram-negative bacteria such as $P$. aeruginosa,
P. vulgaris, E. coli, and A. tumefaciens also showed a higher reduction in CFUs when treated with AgNPs (31 times, 43 times, 28 times, and 13 times more, respectively, compared to $\mathrm{AgNO}_{3}$ ). AgNPs also showed 20 times greater antimicrobial activity compared with $\mathrm{AgNO}_{3}$ in fungal species $C$. albicans. These results pointed out that AgNPs have higher antimicrobial potential against Gram-positive, Gram-negative, and fungal pathogens.

Silver-based products with antimicrobial properties are employed in different industries like medical (eg, antibacterial creams), food (eg, antibacterial agents in packaging), and textiles (eg, preparation of silver wools). ${ }^{32,33}$ The incorporation of silver as nanoparticles has been envisaged as an alternative solution, making these industries more environmentally safe. ${ }^{33}$ The biosynthesized WS AgNPs and WS AgNPs cream showed a strong antimicrobial activity, higher than their $\mathrm{AgNO}_{3}$ counterparts (Figure 7 and Table 2). Silver formulations (gels, creams, etc) are widely used in health sector, namely in wound care. ${ }^{32,33}$ The AgNPs cream produced by the method described in this work might be used for the same purpose with advantage; since it is more
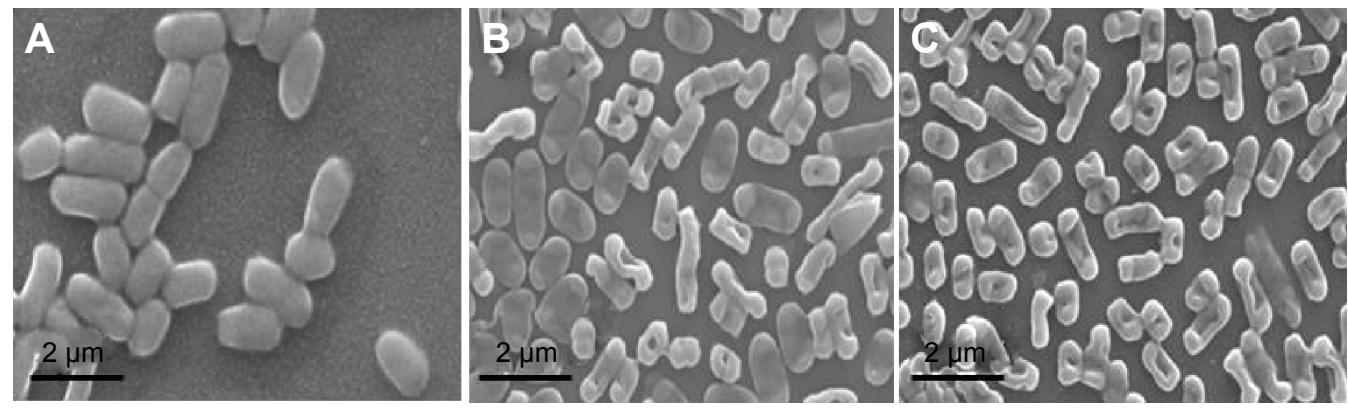

Figure 6 SEM analysis of AgNPs and Escherichia coli interaction.

Notes: Cellular interaction of AgNPs and E. coli visualized by SEM analysis: control (A), after 2 hours (B), and 4 hours (C) incubation time.

Abbreviations: AgNPs, silver nanoparticles; SEM, scanning electron microscopy. 

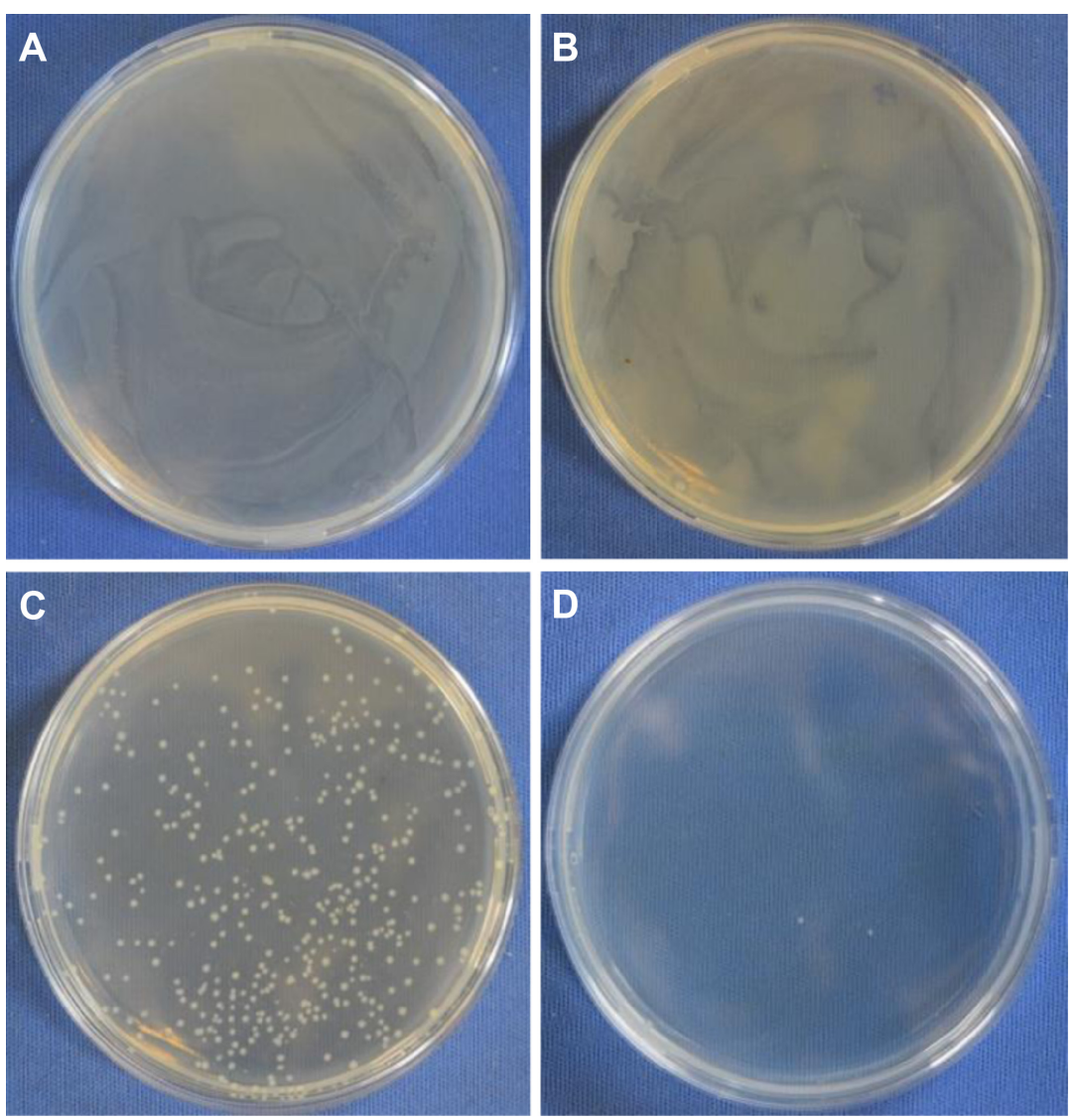

Figure 7 Antibacterial potential of AgNPs by colony counting method.

Notes: Escherichia coli without treatment (just water) (A), E. coli treated with plant extract (B), E. coli treated with $\mathrm{AgNO}_{3}(\mathbf{C})$, and $E$. coli treated with $\mathrm{AgNPs}(\mathbf{D})$. Abbreviation: AgNPs, silver nanoparticles.

effective (antimicrobial), it can be used in lower doses and with less toxicity for the patient, while at the same time being more ecofriendly.

\section{Conclusion}

In conclusion, WS aqueous leaf extracts are suitable for the green synthesis of AgNPs with potent antimicrobial activity. This is highly relevant since the biomass of this plant is considered a waste product by the phytopharmaceutical industry and hence can be used for further economic processes.
Moreover, the results showed that the compounds catechin, p-coumaric acid, luteolin-7-glucoside, and a nonidentified withanolide derivative present in the WS aqueous leaf extract are responsible for green synthesis of AgNPs. The antimicrobial study concludes that AgNPs are 200 times more potent when compared to $\mathrm{AgNO}_{3}$. The AgNPs act by disrupting the cell membrane of $E$. coli, as confirmed by SEM analysis. These AgNPs and cream-incorporated AgNPs have a potential application in many different industries including medical, food, and textiles.

Table 2 Evaluation of antimicrobial potential of AgNPs-incorporated cream

\begin{tabular}{|c|c|c|c|c|}
\hline Organisms & $\begin{array}{l}\text { Water (control) } \\
\text { (CFU/mL) }\end{array}$ & $\begin{array}{l}\text { Empty cream } \\
\text { (CFU/mL) }\end{array}$ & $\begin{array}{l}\mathrm{AgNO}_{3} \text { in cream } \\
(\mathrm{CFU} / \mathrm{mL})\end{array}$ & $\begin{array}{l}\text { AgNPs in cream } \\
(\mathrm{CFU} / \mathrm{mL})\end{array}$ \\
\hline Staphylococcus aureus & $226.3 \pm 0.74$ & $211.0 \pm 0.69 *$ & $123.3 \pm 0.74 * * *$ & $0.6 \pm 0.25 * * *,++$ \\
\hline Pseudomonas aeruginosa & $432.0 \pm 0.69$ & $408.0 \pm 1.00 *$ & $267.6 \pm 0.67 * * *$ & $8.6 \pm 0.48 * * *,++$ \\
\hline Candida albicans & $264.6 \pm 0.88$ & $127.6 \pm 0.75 * * *$ & $33.3 \pm 0.68 * * *$ & $1.6 \pm 0.25 * * *,++$ \\
\hline Proteus vulgaris & $213.0 \pm 1.17$ & $194.6 \pm 1.02$ & $26.6 \pm 0.64 * * *$ & $0.6 \pm 0.25 * * *,++$ \\
\hline Escherichia coli & $471.0 \pm 1.39$ & $441.3 \pm 1.36$ & $226.0 \pm 1.16 * * *$ & $8.0 \pm 0.66 * * *,++$ \\
\hline Agrobacterium tumefaciens & $190.3 \pm 1.47$ & $145.3 \pm 0.88^{*}$ & $35.0 \pm 1.02 * * *$ & $2.66 \pm 0.58 * * *,+$ \\
\hline
\end{tabular}

Notes: Data were analyzed by nonparametric $t$-test using GraphPad Prism 5 software (GraphPad, La Jolla, CA, USA). Data are presented as mean \pm SEM of four replications. Asterisks denote statistically significant difference from water control $(* P<0.05, * * * P<0.001)$. Plus symbols denote statistically significant difference of $A g N P s$ s $\left({ }^{+} P<0.01\right.$, ${ }^{++} \mathrm{P}<0.00 \mathrm{I}$ ) compared with different concentrations of $\mathrm{AgNO}_{3}$.

Abbreviations: AgNPs, silver nanoparticles; SEM, scanning electron microscopy; CFU, colony forming unit. 


\section{Acknowledgments}

This work was supported by Fundação para a Ciência e Tecnologia (FCT), projects (PTDC/AGR-ALI/105169/2008 and PTDC/AGR-GPL/119211/2010). Gregory Marslin is supported by a FCT PhD fellowship (SFRH/BD/72809/2010).

\section{Disclosure}

The authors report no conflicts of interest in this work.

\section{References}

1. Karuppiah M, Rajmohan R. Green synthesis of silver nanoparticles using Ixora coccinea leaves extract. Mater Lett. 2013;97:141-143.

2. Zayed MF, Eisa WH, Shabaka AA. Malva parviflora extract assisted green synthesis of silver nanoparticles. Spectrochim Acta A. 2012;98: 423-428.

3. Jagtap UB, Bapat VA. Green synthesis of silver nanoparticles using Artocarpus heterophyllus Lam. seed extract and its antibacterial activity. Ind Crop Prod. 2013;46:132-137.

4. Tan Y, Wang Y, Jiang L, Zhu D. Thiosalicylic acid-functionalized silver nanoparticles synthesized in one-phase system. J Colloid Interface Sci. 2002;249(2):336-345.

5. Devaux X, Laurent C, Rousset A. Chemical synthesis of metal nanoparticles dispersed in alumina. Nanostruct Mater. 1993;2(4):339-346.

6. Geethalakshmi R, Sarada DVL. Characterization and antimicrobial activity of gold and silver nanoparticles synthesized using saponin isolated from Trianthema decandra L. Ind Crop Prod. 2013;51:107-115.

7. Vijayakumar M, Priya K, Nancy FT, Noorlidah A, Ahmed ABA. Biosynthesis, characterisation and anti-bacterial effect of plant-mediated silver nanoparticles using Artemisia nilagirica. Ind Crop Prod. 2013;41:235-240.

8. Jain D, Daima HK, Kachhwaha S, Kothari SL. Synthesis of plant-mediated silver nanoparticles using papaya fruit extract and evaluation of their anti microbial activities. Dig J Nanomater Bios. 2009;4(3):557-563.

9. Vilchis-Nestor AR, Sanchez-Mendieta V, Carnacho-Lopez MA, Gomez-Espinosa RM, Camacho-Lopez MA, Arenas-Alatorre JA. Solventless synthesis and optical properties of Au and Ag nanoparticles using Camellia sinensis extract. Mater Lett. 2008;62(17-18):3103-3105.

10. Abu Bakar NHH, Ismail J, Abu Bakar M. Synthesis and characterization of silver nanoparticles in natural rubber. Mater Chem Phys. 2007;104(2-3):276-283.

11. Gregory M, Selvakesavan RK, Franklin G, Sarmento B, Dias ACP. Green synthesis of silver nanoparticles using Withania somnifera extract and their incorporation into a cream with antibacterial activity. Planta Med. 2014;80(16):1362-1363.

12. Bhattacharya SK, Muruganandam AV. Adaptogenic activity of Withania somnifera: an experimental study using a rat model of chronic stress. Pharmacol Biochem Be. 2003;75(3):547-555.

13. Mirjalili MH, Moyano E, Bonfill M, Cusido RM, Palazon J. Steroidal lactones from Withania somnifera, an ancient plant for novel medicine. Molecules. 2009;14(7):2373-2393.

14. Gregory M, Sarmento B, Dias A. Nanoencapsulation of a Withania somnifera extract with PCL and MPEG-PCL di-block copolymer. Planta Med. 2014;80(16):1467-1468.
15. Rajaswara Rao BR, Rajpu DK, Nagaraju G, Aainarayana G. Opportunities and challenges in the cultivation of ashwaganda (Withania somnifera (1.) Dunal). J Pharmacogn. 2012;3(2):88-91.

16. Marslin G, Sheeba CJ, Kalaichelvan VK, Manavalan R, Reddy PN, Franklin G. Poly(D,L-lactic-co-glycolic acid) nanoencapsulation reduces Erlotinib-induced subacute toxicity in rat. J Biomed Nanotechnol. 2009;5(5):464-471.

17. Gregory M, Sarmento B, Duarte S, et al. Curcumin loaded MPEG-PCL di-block copolymer nanoparticles protect glioma cells from oxidative damage. Planta Med. 2014;80(16):1468-1468.

18. Dias A, Seabra R, Andrade P, Fernandes-Ferreira M. the development and evaluation of an hplc-dad method for the analysis of the phenolic fractions from in vivo and in vitro biomass of hypericum species. J Liq Chromatogr Related Technol. 1999;22(2):215-227.

19. Raut RW, Mendhulkar VD, Kashid SB. Photosensitized synthesis of silver nanoparticles using Withania somnifera leaf powder and silver nitrate. J Photoch Photobio B. 2014;132:45-55.

20. Joint Committee on Powder Diffraction standard (JCPDS) Data Cord No -04-0783. Power Diffraction File. The Centure publisher; 1991

21. Amutha M, Lalitha P, Firdhouse MJ. Biosynthesis of silver nanoparticles using Kedrostis foetidissima (Jacq.) Cogn. J Nanotechnol. 2014;2014:5.

22. Tamuly C, Hazarika M, Borah SC, Das MR, Boruah MP. In situ biosynthesis of Ag, Au and bimetallic nanoparticles using Piper pedicellatum C.DC: green chemistry approach. Colloid Surface B. 2013;102:627-634.

23. Nadagouda MN, Varma RS. Green synthesis of silver and palladium nanoparticles at room temperature using coffee and tea extract. Green Chem. 2008;10(8):859-862.

24. Santos SAO, Pinto RJB, Rocha SM, et al. Unveiling the chemistry behind the green synthesis of metal nanoparticles. Chemsuschem. 2014;7(9):2704-2711.

25. Lok CN, Ho CM, Chen R, et al. Proteomic analysis of the mode of antibacterial action of silver nanoparticles. J Proteome Res. 2006;5(4):916-924.

26. Velusamy P, Das J, Pachaiappan R, Vaseeharan B, Pandian K. Greener approach for synthesis of antibacterial silver nanoparticles using aqueous solution of neem gum (Azadirachta indica L.). Ind Crop Prod. 2015;66:103-109.

27. Mukherjee S, Chowdhury D, Kotcherlakota R, et al. Potential theranostics application of bio-synthesized silver nanoparticles (4-in-1 system) Theranostics. 2014;4(3):316-335.

28. Dror-Ehre A, Mamane H, Belenkova T, Markovich G, Adin A. Silver nanoparticle- $E$. coli colloidal interaction in water and effect on $E$-coli survival. J Colloid Interface Sci. 2009;339(2):521-526.

29. Zhang YW, Peng HS, Huang W, Zhou YF, Yan DY. Facile preparation and characterization of highly antimicrobial colloid Ag or Au nanoparticles. J Colloid Interface Sci. 2008;325(2):371-376.

30. Cushnie TP, Lamb AJ. Recent advances in understanding the antibacterial properties of flavonoids. Int J Antimicrob Ag. 2011;38(2):99-107.

31. Daglia M. Polyphenols as antimicrobial agents. Curr Opin Biotech. 2012;23(2):174-181.

32. Chopra I. The increasing use of silver-based products as antimicrobial agents: a useful development or a cause for concern? J Antimicrob Chemother. 2007;59(4):587-590.

33. Rai M, Yadav A, Gade A. Silver nanoparticles as a new generation of antimicrobials. Biotechnol Adv. 2009;27(1):76-83.
International Journal of Nanomedicine

\section{Publish your work in this journal}

The International Journal of Nanomedicine is an international, peerreviewed journal focusing on the application of nanotechnology in diagnostics, therapeutics, and drug delivery systems throughout the biomedical field. This journal is indexed on PubMed Central, MedLine, CAS, SciSearch ${ }^{\circledR}$, Current Contents ${ } /$ Clinical Medicine,

\section{Dovepress}

Journal Citation Reports/Science Edition, EMBase, Scopus and the Elsevier Bibliographic databases. The manuscript management system is completely online and includes a very quick and fair peer-review system, which is all easy to use. Visit http://www.dovepress.com/ testimonials.php to read real quotes from published authors. 